BNL-73446-2005-CP

\title{
Stochastic Cooling for Bunched Beams
}

\author{
M. Blaskiewicz \\ Presented at the Particle Accelerator Conference (PAC'05) \\ Knoxville, Tennessee \\ May 16-20, 2005
}

Collider-Accelerator Department

Brookhaven National Laboratory

P.O. Box 5000

Upton, NY 11973-5000

wWw.bnl.gov

Managed by

Brookhaven Science Associates, LLC

for the United States Department of Energy under

Contract No. DE-AC02-98CH10886

This is a preprint of a paper intended for publication in a journal or proceedings. Since changes may be made before publication, this preprint is made available with the understanding that it will not be cited or reproduced without the permission of the author. 


\section{DISCLAIMER}

This report was prepared as an account of work sponsored by an agency of the United States Government. Neither the United States Government nor any agency thereof, nor any of their employees, nor any of their contractors, subcontractors, or their employees, makes any warranty, express or implied, or assumes any legal liability or responsibility for the accuracy, completeness, or any third party's use or the results of such use of any information, apparatus, product, or process disclosed, or represents that its use would not infringe privately owned rights. Reference herein to any specific commercial product, process, or service by trade name, trademark, manufacturer, or otherwise, does not necessarily constitute or imply its endorsement, recommendation, or favoring by the United States Government or any agency thereof or its contractors or subcontractors. The views and opinions of authors expressed herein do not necessarily state or reflect those of the United States Government or any agency thereof.

FOR UNCLASSIFIED, UNLIMITED STI PRODUCTS

Available electronically at:

OSTI:

http://www.osti.gov/bridge

Available for a processing fee to U.S. Department of Energy and its contractors, in paper from:

U.S. Department of Energy

Office of Scientific and Technical Information

P.O. Box 62

Oak Ridge, TN 37831

Phone: (865) 576-8401

Facsimile: (865) 576-5728

E-mail: reports@adonis.osti.gov

National Technical Information Service (NTIS):

Available for sale to the public from:

U.S. Department of Commerce

National Technical Information Service

5285 Port Royal Road

Springfield, VA 22131

Phone: (800) 553-6847

Facsimile: (703) 605-6900

Online ordering: http://www.ntis.gov/ordering.htm

(3) Fintent an raspeled paplat 


\title{
Stochastic Cooling for Bunched Beams*
}

\author{
M. Blaskiewicz ${ }^{\dagger}$ BNL 911B, Upton, NY 11973, USA
}

\section{Abstract}

Problems associated with bunched beam stochastic cooling are reviewed. A longitudinal stochastic cooling system for RHIC is under construction and has been partially commissioned. The state of the system and future plans are discussed.

\section{INTRODUCTION}

Bunched beam stochastic cooling was observed in 1978 in ICE [1]. A theory was developed in the early eighties [2, $3,4]$ and stochastic cooling in the SPS [5, 6], RHIC [7, 8] and the Tevatron $[5,9]$ was explored. For these colliders the particle densities were much higher than those in ICE and early on $[9,10,11,12,13]$ it was found that "RF activity" extending up to very high frequencies swamped the true Schottky signal. It was suspected that these signals could be due to coherent oscillations or sharp edge phase space distributions. Systematic studies in RHIC $[14,15,16]$ have verified the existence of various kinds of coherent signals. For ions these problems are manageable and a longitudinal stochastic cooling system is under construction. The paper begins with a discussion of these unexpected signals.

\section{SOURCES OF "RF ACTIVITY"}

In RHIC we have seen two fundamentally different types of strong coherent signals. The simplest is the coherent signal observed with heavy ion beams. For heavy ions the bunches are injected with Lorentz factor $\gamma \approx 11$ into $28 \mathrm{MHz}$ buckets with harmonic number $h=360$. The bunches are accelerated through transition $\left(\gamma_{t}=22.8\right)$ to top energy with $\gamma \approx 105$. After reaching top energy the particles are rebucketed. The energy-time phase space evolution of this process is shown in Figure 1.

The transfer of the bunch from $h=360$ to $h=2520$ is never perfect, as shown in Figure 1.f. Consider the situation after the RF gymnastics are finished. Neglect coherent forces and use the smooth approximation. With phase $\phi$ and energy variable $p=d \phi / d t$ the dynamics are generated by a Hamiltonian $H(p \phi)=p^{2} / 2+U(\phi)$. Let $\Psi(p, \phi, t)$ be the coarse grained phase space density. This density evolves and filaments in complicated ways. Consider Figure 2. There are 12 regions bounded by separatricies and, since $d H / d t=0$ for a given particle, the probability distribution for the energy in each region $k$ is independent of

\footnotetext{
* Work performed under the auspices of the United States Department of Energy.

† blaskiewicz@bnl.gov
}
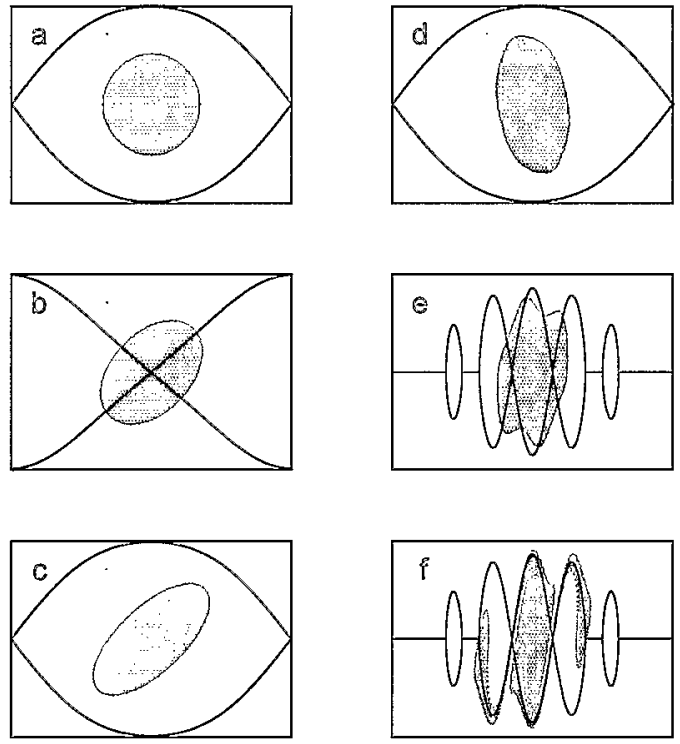

Figure 1: Schematic of the RHIC rebucketing process showing the particles (red) and some instantaneous RF trajectories (blue). Initially (a) the bunches are matched in the $28 \mathrm{MHz}(h=360)$ buckets. The RF phase is shifted by $180^{\circ}$ and the bunch dilates along the separatrix (b). The RF phase is shifted back and the bunch undergoes about $3 / 8$ of a synchrotron oscillation until reaching minumum length, (c) and (d). The $197 \mathrm{MHz}$ system $(h=2520)$ comes on trapping the shortened bunch, (e) and (f).

time

$$
P_{k}(H)=\int_{\text {regionk }} d p d \phi \Psi(p, \phi, t) \delta\left[H-p^{2} / 2-U(\phi)\right]
$$

In each of the regions we assume the existence of action angle variables $J_{k}, \Psi_{k}$ with $d J_{k} d \Psi_{k}=d p d \phi$. By definition $d H / d J_{k}=2 \pi / T_{k}(H)$ with $T_{k}(H)$ being the oscillation period as a function of energy in region $k$. Therefore, after the beam is fully filamented the distribution function in region $k$ is given by $\Psi_{k}(p, \phi)=P_{k}[H(p, \phi)] / T_{k}[H(p, \phi)]$. This can result in discontinuities in the phase space density and almost always results in strongly discontinuous derivatives at the separatricies. Integrating over the energy yields the instantaneous bunch current and the phase space features manifest as discontinuities in the derivative of the bunch current. For bunches that start out roughly equal, each bunch will end up with similar discontinuities. These discontinuities are locked with respect to the RF so the bunches add power coherently and the spectral envelope depends on the fill pattern. This has been observed 


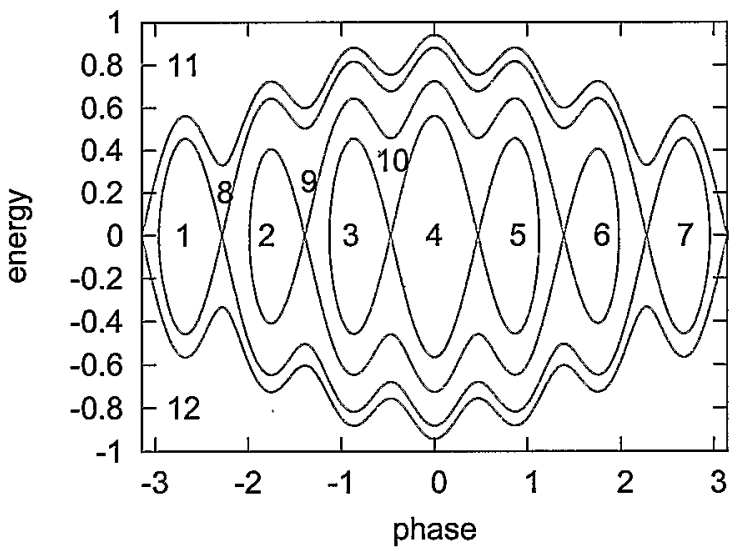

Figure 2: Separatricies for simultaneous voltage at $h=360$ and $h=2520$. The 12 regions bounded by separatricies are shown. For clarity, the ratio of the storage to accleration voltages was about $1 / 4$ that normally used.

using a spectrum analyzer with gold beams $[16,17]$. Figure 3 shows time domain signals for five different copper bunches obtained with the FNAL pickup [18] and an oscilloscope graciously loaned by Agilent. Some low level processing, described in the section on RHIC cooling, was applied and the signal was transmitted using a fiber-optic link. The $40 \mathrm{GHz}$ sampling rate and $20 \mathrm{GHz}$ analog bandwidth was more than sufficient for sampling the $4-8 \mathrm{GHz}$ signal. As one can see, the average of the five signals is close in magnitude to any given signal. The time between the acquisition of the different traces was of order one minute, so the average is locked to the RF. This confirms previous work using spectra and one concludes that the coherent signals for ions are associated with sharp features in the bunch profile and that these sharp features are associated with the RF.

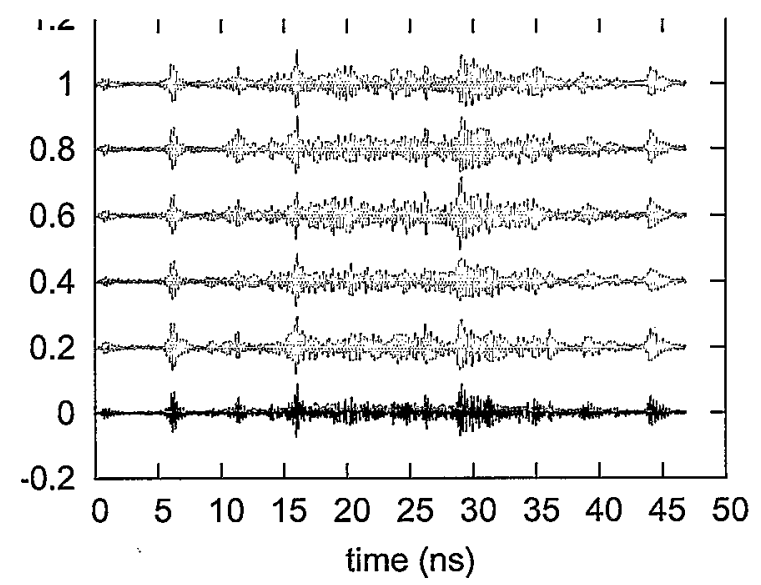

Figure 3: Time domain signals for five different copper bunches (red). The signals were shifted to align the minimum near $7 \mathrm{~ns}$. The averge of the 5 (blue) and the standard deviation (orange) are also shown.
For spin polarized protons the $h=2520$ system is on at a low level throughout the ramp and turned up slowly at store. During 2004 there was a significant amount of power at each revolution line and the power varied by about $5 \mathrm{~dB}$ due to the non-uniform fill pattern. A comparable spectrum from May 2005 showed strong features associated with the $4.68 \mathrm{MHz}$ bunching frequency and the abort gap suggesting a situation similar to that for heavy ions. For both cases a strong narrow band signal dominates the power. At times it appears that the narrow band signal is simply a revolution harmonic but at others a significant amount of power is present in a few synchrotron sidebands. The signals away from the revolution line, which dominated the spectra in 2004 , might be due to solitary waves [19] or something currently unknown. For the heavy ion beams in RHIC the coherent lines are less complicated and we go on to discuss the cooling system proper.

\section{PRELIMINARY CONSIDERATIONS}

Before discussing technical details it is appropriate to review the underlying physics.Coasting beam theory is reviewed in [20]. Bunched beam theory was developed by Chattophadhyay $[2,3,4]$ and applied to RHIC by Wei and Ruggerio $[8,21]$. One begins by defining the azimuth $\theta$ which increases by $2 \pi$ each turn and $\omega_{0}$, the angular revolution frequency of a synchronous particle. The phase of a given particle is $\phi=\theta-\omega_{0} t$. The bunched beam theory is based upon the approximation that the unperturbed phase of a particle can be described by $\phi(t)=$ $a \sin \left[\omega_{s}(a) t+\psi_{0}\right]$, where $a$ is the amplitude of the oscillation, $\omega_{s}(a)$ is the amplitude dependent angular synchrotron frequency and $\psi_{0}$ is the initial phase. Stochastic cooling is a form of feedback so the beam transfer function (BTF) determines the maximum stable gain and, via signal suppression, allows for adjustment of the cooling system. Therefore, let the stochastic cooling kicker apply a voltage $V_{K}(t)=V_{K} \exp (-i \tilde{\omega} t+\epsilon t)$ where the kicker is located at azimuth $\theta_{K}$ and a small positive $\epsilon$ has been included to insure causality. Define $\omega=\omega_{0}+d \phi / d t$ so that the change in frequency due to the kicker is

$$
\left.\frac{d \omega}{d t}\right|_{K}=\frac{-\eta \omega_{0}^{2}}{\beta^{2} E_{T} / q} \delta_{p}\left(\theta-\theta_{K}\right) V_{K} \exp (-i \tilde{\omega} t+\epsilon t),
$$

where $\eta=1 / \gamma_{t}^{2}-1 / \gamma^{2}$ is the slip factor, $\beta=v / c, E_{T}$ and $q$ are the total energy and charge of an ion, and

$$
\delta_{p}(\theta)=\sum_{m=-\infty}^{\infty} \frac{\exp (i m \theta)}{2 \pi}
$$

is the periodic delta function. Let $\omega_{s 0}$ be the small amplitude angular synchrotron frequency and define action angle variables $J$ and $\psi$ via $\phi=\sqrt{2 J / \omega_{80}} \sin \psi$ so that $d \phi d \omega=d J d \psi$. Let $\Psi_{0}(J)$ be the unperturbed distribution. Assume that $V_{K}$ is small and generates a perturbation $\Psi_{1}(J, \psi, t)$. The Vlasov equation in first order perturbation 
theory is

$$
\frac{\partial \Psi_{1}}{\partial t}+\omega_{s}(J) \frac{\partial \Psi_{1}}{\partial \psi}=-\left.\frac{d \omega}{d t}\right|_{K} \sqrt{\frac{2 J}{\omega_{s 0}}} \cos \psi \frac{d \Psi_{0}}{d J} .
$$

Insert the expansion for the periodic delta function and set

$$
\Psi_{1}(J, \psi, t)=\sum_{m} \hat{\Psi}_{m}(J, \Psi) e^{\left[\epsilon-i\left(\tilde{\omega}-m \omega_{0}\right)\right] t-i m \theta_{K}},
$$

so that

$$
\left[\epsilon-i\left(\tilde{\omega}-m \omega_{0}\right)\right] \hat{\Psi}_{m}+\omega_{s}(J) \frac{\partial \hat{\Psi}_{m}}{\partial \psi}=\hat{F}_{m}(J, \psi)
$$

with

$$
\hat{F}_{m}(J, \psi)=V_{K} \frac{\eta \omega_{0}^{2}}{\beta^{2} E_{T} / q} \sqrt{\frac{2 J}{\omega_{s 0}}} \cos \psi e^{i m \phi(J, \psi)} \frac{d \Psi_{0}}{d J} .
$$

Setting $\lambda_{m}=\left[\epsilon-i\left(\tilde{\omega}-m \omega_{0}\right)\right]$ and integrating eq (5) yields

$$
\hat{\Psi}_{m}(J, \Psi)=\int_{0}^{2 \pi} d \psi_{1} \frac{e^{\lambda_{m} \psi_{1} / \omega_{s}(J)} \hat{F}_{m}\left(J, \psi_{1}+\psi\right)}{\omega_{s}(J)\left(e^{2 \pi \lambda_{m} / \omega_{s}(J)}-1\right)} .
$$

The current at the pickup is

$$
I\left(\theta_{P}, t\right)=\omega_{0} q \int d J d \psi \delta_{p}\left(\theta(J, \psi, t)-\theta_{P}\right) \Psi_{1}(J, \psi, t)
$$

where $\Psi$ is normalized to the number of particles. Equation (8) contains frequencies $\tilde{\omega}+n \omega_{0}$. Using the Fourier expansion of the periodic delta function and taking only $n=0$ gives

$$
\begin{aligned}
I\left(\theta_{P}, \tilde{\omega}\right) & =\frac{\omega_{0} q}{2 \pi} \sum_{m} e^{i m\left(\theta_{P}-\theta_{K}\right)} I_{m}(\tilde{\omega}) \\
I_{m}(\tilde{\omega}) & =\int d J d \psi e^{-i m \phi(J, \psi)} \hat{\Psi}_{m}(J, \psi) .
\end{aligned}
$$

Using $\exp (i x \sin \psi)=\sum_{m} J_{m}(x) \exp (i m \psi)$, equation (10) can be reduced to an infinite sum of one dimensional integrals,

$$
\begin{aligned}
I_{m}(\tilde{\omega}) & =\frac{2 \pi}{m} \frac{\eta \omega_{0}^{2}}{\beta^{2} E_{T} / q} \int d J \frac{d \Psi_{0}}{d J} \sum_{n} Q_{n} \\
Q_{n} & =J_{n}^{2}\left(m \sqrt{2 J / \omega_{s 0}}\right) \frac{n}{\lambda_{m}+i n \omega_{s}(J)}
\end{aligned}
$$

Figure 4 shows measured and calculated beam transfer functions for protons. The IF bandwidth for the measurement was $10 \mathrm{~Hz}$ and the model used $\epsilon=20 \mathrm{~s}^{-1}$ which gives a $10 \mathrm{~Hz}$ equivalent width for the power spectrum. The "fuzz" is actually a forest of synchroton lines and the gross feaures of the two BTFs are similar. The black line is the BTF calculated for a coasting beam with the same momentum distribution as was used in the bunched beam calculation [16].

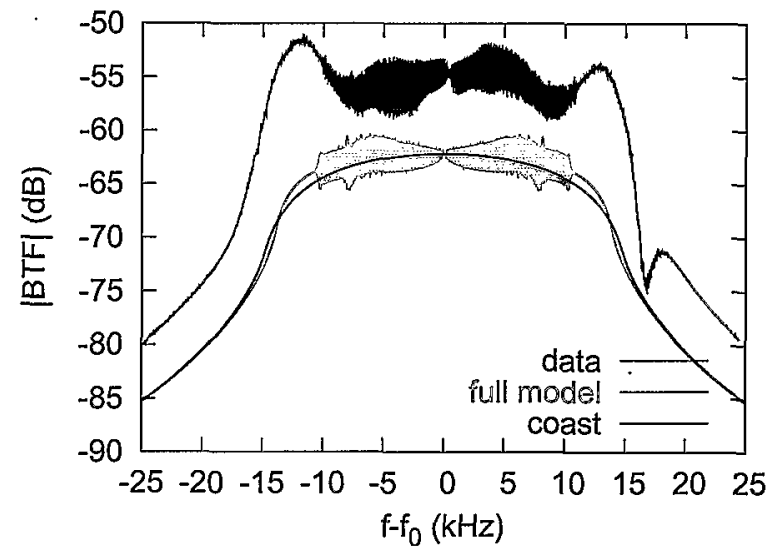

Figure 4: Beam transfer functions for protons near $7.8 \mathrm{Ghz}$. The measurement is blue. The bunched beam calculation is red, and the coasting beam calculation is black. The absolute gain is not calibrated.

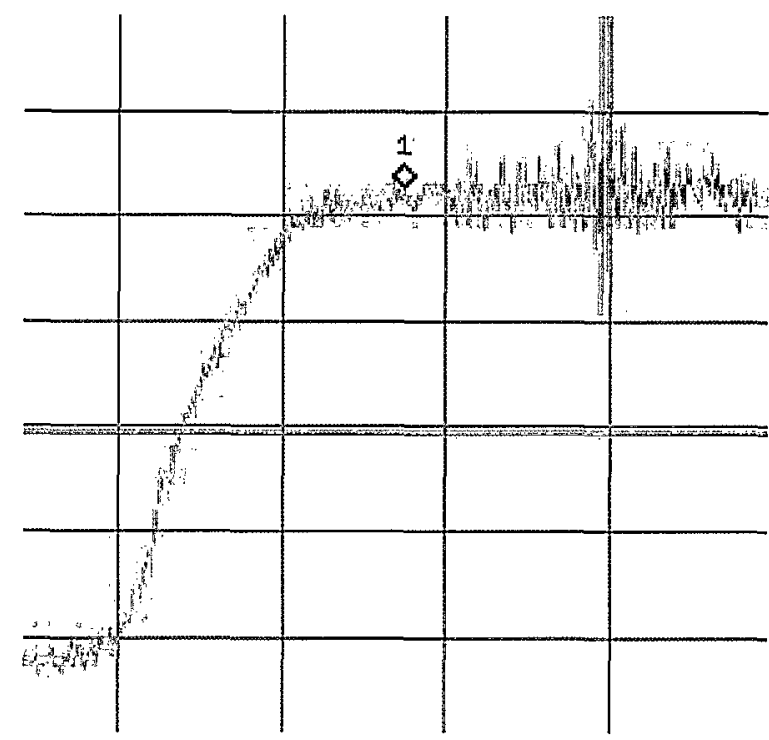

Figure 5: Gold Schottkty spectrum near $5 \mathrm{GHz}$, with $5 \mathrm{kHz}$ per horizontal box, $5 \mathrm{~dB}$ per vertical box, and $30 \mathrm{~Hz}$ resolution bandwidth. The synchrotron frequency was $\sim 200 \mathrm{~Hz}$ so fluctuations in the spectrum are resolved.

For the curves in Figure 4 the bunch length was less than half the length of the RF bucket. This is necessary for the approximation $\phi=a \sin \psi$ to hold accurately, but leads to a small synchrotron frequency spread. When cooling heavy ions the RF bucket is overfull and the Schottky spectrum is quite smooth, as shown in Figure 5. Also notice that any prominent synchrotron lines occur near the revolution line and will be strongly suppressed for longitudinal filter cooling. Therefore, we assume that the beam response to the cooling system will be comparable to that for a coasting beam, albeit with modulations associated with bunching. 


\section{LONGITUDINAL FILTER COOLING IN RHIC}

For RHIC the main purpose of the cooling system is to counteract intrabeam scattering (IBS) and keep the beam in the RF buckets. To keep costs down the signal between the pickup and the kicker travels within the tunnel in the direction opposite the beam. The revolution period is $T_{0}=$ $12.8 \mu \mathrm{s}$. For a fiber optic transmission line this limited us to a delay $2 / 3$ of a turn or $T_{d}=8.5 \mu$ s between pickup an kicker. At this point we have worked on the yellow (counterclockwise) ring. The pickup is in the $120^{\prime}$ clock straight section and the kicker is in the $4 o^{\prime}$ 'clock straight section. We plan to cool at energies well above transition so most of the phase slip is generated in the arcs and the effective delay is very close to $2 / 3$ of a turn. For gold with $\gamma=107$ and $4 \mathrm{MV}$ at $h=2520$ the frequency spread at the edge of the bucket is $\left(\omega-\omega_{0}\right) / \omega_{0}= \pm 2.8 \times 10^{-6}$. For a one turn filter cooling system the transfer function is $G_{1}(f)=\left[1-\exp \left(i 2 \pi \Delta f T_{0}\right)\right] \exp \left(i 2 \pi \Delta f T_{d}\right)$ with $\Delta f$ the difference between the drive frequency and the nearest revolution line. The imaginary part of $G_{1}(f)$ is antisymmetric about a revolution line as is needed for cooling. The gain is correct as long as $|\Delta f| \leq 16.5 \mathrm{kHz}$. With the frequency spread in RHIC this limits a one turn delay cooling system to an upper frequency of $5.9 \mathrm{GHz}$. Now consider $G_{2}(f)=G_{1}(f)\left[1-\exp \left(i 2 \pi \Delta f T_{0}\right)\right]$, which is two one turn delay notch filters in series. With this filter the gain has the right sign for $|\Delta f| \leq 23.4 \mathrm{kHz}$ corresponding to an upper frequency of $8.3 \mathrm{GHz}$. The RHIC design used $G_{2}$ and the upper frequency of the cooling system is $8 \mathrm{GHz}$. For the fall run of 2005 we plan on having a lower frequency of $5 \mathrm{Ghz}$.

To estimate system performance we take a coasting beam approximation. For cooling that is slow compared with the revolution frequency the density obeys a damped diffusion equation $[22,20,23]$. Take $\Psi(\omega) d \omega$ to be the fraction of particles with revolution frequencies in $d \omega$ then

$$
\frac{\partial \Psi}{\partial t}=\frac{\partial}{\partial \omega}\left[-F(\omega) \Psi(\omega, t)+D(\omega, t) \frac{\partial \Psi}{\partial \omega}\right] .
$$

The drift and diffusion coefficients are

$$
\begin{aligned}
& F(\omega)=\tilde{C} \sum_{m=-\infty}^{\infty} e^{i m\left[\theta_{P}-\theta_{K}\right] \omega Z_{D}[m \omega]} \\
& D(\omega)=\pi N \tilde{C}^{2} \sum_{m=-\infty}^{\infty} \omega^{2}\left|Z_{D}(m \omega)\right|^{2} \frac{\Psi(\omega)}{|m|} .
\end{aligned}
$$

where $\tilde{C}=\eta q^{2} /\left(T_{0}^{2} \beta^{2} E_{T}\right), N$ is the number of ions in the ring and $Z_{D}(\omega)$ is the "dressed" transfer impedance

$$
Z_{D}(\tilde{\omega})=\frac{Z_{T}(\tilde{\omega})}{1+Z_{T}(\tilde{\omega}) Y_{b} B(\tilde{\omega})},
$$

where $Z_{T}(\tilde{\omega})=-V_{K} / I\left(\theta_{P}, \tilde{\omega}\right)$ is the low gain transfer impedance and

$$
Y_{b}=\frac{N q^{2} \omega_{0} \eta}{4 \pi^{2} \beta^{2} E_{0}}
$$

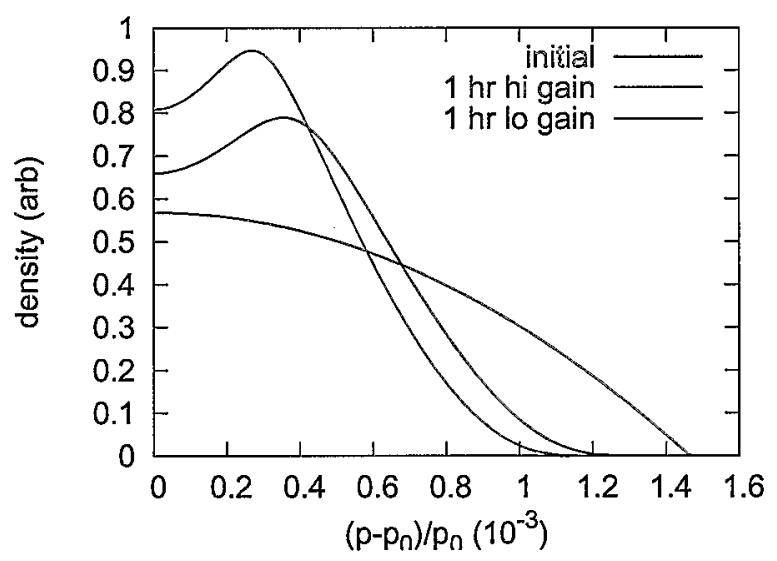

Figure 6: Stochastic cooling simulations for $10^{9}$ gold ions in a $5 \mathrm{~ns}$ long bunch.

The BTF for a coasting beam is

$$
B(\tilde{\omega})=\omega_{0}^{2} \sum_{k=-\infty}^{\infty} \lim _{\epsilon \rightarrow 0^{+}} \int d \omega \frac{e^{i k\left(\theta_{P}-\theta_{K}\right)}}{\epsilon+i(k \omega-\tilde{\omega})} \frac{d \Psi(\omega)}{d \omega} .
$$

Numerical integrations of these equations for RHIC conditions are shown in Figure 6. The curve labeled "hi gain" corresponds to a system gain that is close to the stability threshold implied by eq (16). This gain was halved and the resulting distribution after an hour is labeled "lo . gain". These simulations also included a reasonably accurate model of IBS [23].

To generate the necessary voltage notice that the central part of the bunch is only $5 \mathrm{~ns}$ long while the bunch spacing is $100 \mathrm{~ns}$. By using cavity kickers with resonant frequencies $5,5.2, \ldots 7.8,8.0 \mathrm{GHz}$ one can use Fourier decomposition to obtain the correct voltage at each bunch passage $[1,10]$. A full width half power bandwidth of $10 \mathrm{MHz}$ allows the cavities to change amplitude and phase between bunch passages. For a cavity with $R / Q=100 \Omega, 40$ Watts of amplifier power yields an rms voltage of $1.6 \mathrm{kV}$ at $6.5 \mathrm{GHz}$. Both simulations and order of magnitude calculations show this is an acceptable voltage. To drive the cavities we use a traversal filter in series with $G_{2}$. Taking a delay between the filter branches of $5 \mathrm{~ns}$ and using 16 branches one obtains a piecewice periodic drive signal. Additional filters of $100 \mathrm{MHz}$ bandwidth remove unwanted frequencies. To stop saturation a traversal filter is applied in the tunnel before the fiber-optic transmitter. A 5 branch filter was present for the data in Figure 3. Much work went into avoiding saturation from the coherent signals of Figure 3.

The $\mathrm{TM}_{0,1,0}$ mode cutoff radius at $8 \mathrm{GHz}$ is $1.4 \mathrm{~cm}$ and we took a pipe radius of $1 \mathrm{~cm}$ for the cavities. To reduce aperture limitations during injection and acceleration the kicker cavities are split along the beam axis and are closed only after reaching flattop. The tanks and motors were supplied by FNAL and retrofitted for our application. During 


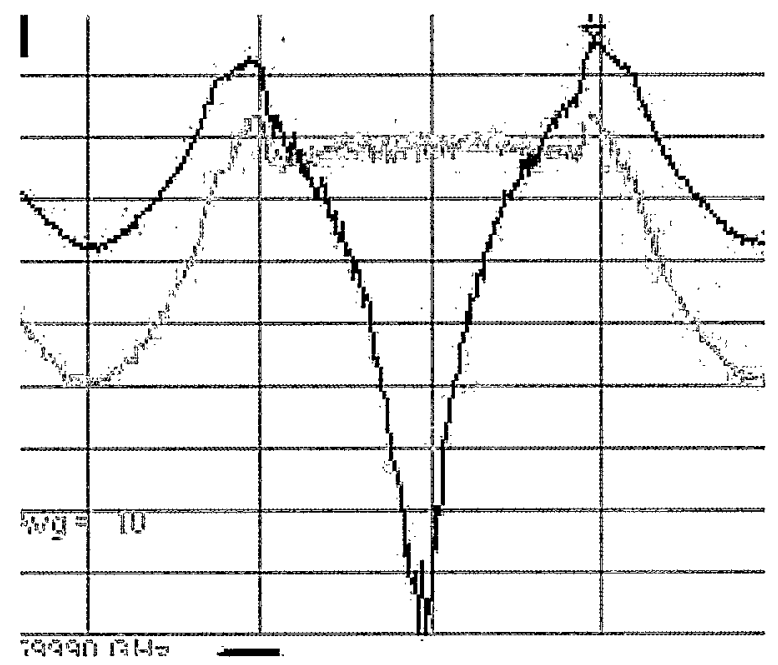

Figure 7: Magnitude of the BTF for copper at $\gamma=107$ with and without filter $G_{2}$. The center frequency was $7.6 \mathrm{GHz}$ and there are $20 \mathrm{kHz}$ per horizontal box and $5 \mathrm{~dB}$ per vertical division.

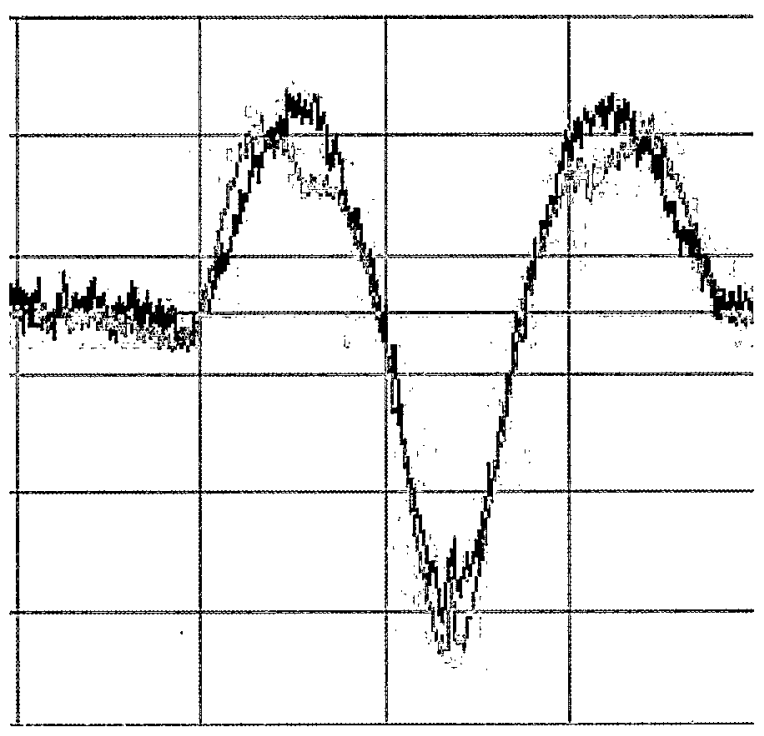

Figure 8: Signal suppression for copper beam at $7.8 \mathrm{GHz}$, there are $20 \mathrm{kHz}$ per horizontal division and $5 \mathrm{~dB}$ per vertical division.

the spring of 2005 there were 12 cavities installed, spanning the $4-5 \mathrm{GHz}$ and $7-8 \mathrm{GHz}$ bands. Figure 7 shows the open loop beam transfer function for copper beams at top energy. The filter produces a good notch at the revolution line and the real and imaginary parts of the BTF have appropriate symmetry. Signal suppression for this configuration is shown in Figure 8. Full cooling in the yellow ring is planned for this fall.

\section{ACKNOWLEDGEMENTS}

This work would have been impossible without the dedication and expertise of my primary collaborator, J.M. Brennan. On the theory side, Jie Wei provided valueable insights. Dave Mcginnis and Ralph Pasquinelli of FNAL, helped with both the hardware, and our understanding of how to use it. Many thanks to Fritz Caspers and Flemming Pedersen of CERN for useful conversations and encouragement. Finally, I acknowledge the dedication and expertise of the BNL RF and instrumentation group.

\section{REFERENCES}

[1] H. Herr, D. Mohl, Porc. Workshop on Phase Space Cooling of High Energy Beams, Madison WI, USA, 1978. also, CERN/EP/Note 79-34 (1979), and CERN/PS/DL/Note 79-3 (1979).

[2] S. Chattopadhyay, LBL-14826, (1982).

[3] S. Chattopadhyay, IEEE TNS 30, No. 4, p2646, 1983.

[4] S. Chattopadhyay, IEEE TNS 30, No. 4, p2649, 1983.

[5] S. Chattopadhyay, IEEE TNS 30, No. 4, p2334, 1983.

[6] D. Boussard, S. Chattopadhyay, G. Dome, T. Linnecar, CERN 84-15, p197, (1984).

[7] S. Van de Meer, RHIC-AP-9 (1984).

[8] J. Wei, A.G. Ruggiero, BNL/AD/RHIC-71 (1990).

[9] G. Jackson, PAC91, p2532, 1991.

[10] D. Boussard, Proc. Joint US-CERN Acc. School, Texas, 1986, Lecture Notes on Phys. 296, p289

[11] G. Jackson, J. Marriner, D. McGinnis, R. Pasquinelli, D. Peterson, Workshop on Advanced Beam Instrumentation, Tsukuba, p312, (1991).

[12] F. Caspers, D. Mohl, XVII International Conference on High Energy Accelerators, Dubna, Russia, (1998). also CERN/PS 98-051 (DI).

[13] G. Jackson, Workshop on Beam Cooling, Montreux, 1993, CERN 94-03, (1994).

[14] J.M. Brennan, M. Blaskiewicz, P. Cameron, J. Wei, 308, EPAC02

[15] M. Blaskiewicz, J.M. Brennan, P. Cameron, J. Wei, PAC03, p394.

[16] M. Blaskiewicz, J.M. Brennan, J. Wei, EPAC04, p2861, (2004).

[17] J.M. Brennan, M. Blaskiewicz, J. Wei, NIMA, 532, p335, (2004)

[18] R.J. Pasquinelli, PAC95 Dallas, p2379 (1995).

[19] M. Blaskiewicz, J. Wei, A. Luque, H. Schamel, PRSTAB, 7, 044402 (2004)

[20] D. Mohl, CERN 87-03, p 453 (1987).

[21] J. Wei, Workshop on Beam Cooling, Montreux, 1993, CERN 94-03, (1994), and these proceedings.

[22] J. Bisognano, C. Leemann, AIP conf 87, p583, AIP New York, (1982).

[23] M. Blaskiewicz, C-AD note \#189 (2005). 